\title{
Scenario reduction in stochastic programming with respect to discrepancy distances
}

\author{
René Henrion ${ }^{1}$, Christian Küchler ${ }^{2}$ and Werner Römisch ${ }^{2}$ \\ ${ }^{1}$ Weierstrass Institute for Applied Analysis and Stochastics, 10117 Berlin, Germany \\ ${ }^{2}$ Humboldt-University Berlin, Institute of Mathematics, 10099 Berlin, Germany
}

\begin{abstract}
Discrete approximations to chance constrained and mixed-integer two-stage stochastic programs require moderately sized scenario sets. The relevant distances of (multivariate) probability distributions for deriving quantitative stability results for such stochastic programs are $\mathcal{B}$-discrepancies, where the class $\mathcal{B}$ of Borel sets depends on their structural properties. Hence, the optimal scenario reduction problem for such models is stated with respect to $\mathcal{B}$-discrepancies. In this paper, upper and lower bounds, and some explicit solutions for optimal scenario reduction problems are derived. In addition, we develop heuristic algorithms for determining nearly optimally reduced probability measures, discuss the case of the cell discrepancy (or Kolmogorov metric) in some detail and provide some numerical experience.
\end{abstract}

Keywords: Stochastic programming, chance constraints, two-stage, mixed-integer, scenario reduction, discrepancy, Kolmogorov metric.

2000 MSC: $90 \mathrm{C} 15$

\section{Introduction}

Quantitative stability studies in stochastic programming (see [18] for a recent survey) indicate which probability metrics (i.e., distances of probability distributions) are relevant and, in some sense, even canonical for the stability of specific classes of stochastic programs. In particular, Fortet-Mourier and Wasserstein metrics are relevant for two-stage stochastic programs [19, 17], $\mathcal{B}$-discrepancies are canonical for chance constrained models $[20,7,8]$ and also relevant for two-stage mixed-integer models $[22,17]$. The class $\mathcal{B}$ of Borel sets is chosen as small as possible but large enough to contain all sets appearing in chance constrained models or all closures of continuity regions of integrands in two-stage mixed-integer models.

Fortet-Mourier metrics have recently been used for the (nearly) optimal reduction of discrete probability distributions in two-stage stochastic programs without integrality requirements $[3,4,6]$. Furthermore, a multiperiod recursive extension of the reduction technique is developed in [5] for generating scenario trees as inputs of multistage stochastic 
programs. Inserting discrete probability distributions into chance constrained and twostage mixed-integer stochastic programs represents an often used approximation technique for solving these models (cf. [21]). Such discrete approximations lead to mixed-integer programs in both cases (see [15, Chapter 11.9] for chance constrained models), whose dimensions grow rapidly with the number of scenarios involved. Hence, moderately sized scenario sets that represent good approximations of the underlying probability distribution are even of greater importance than for two-stage models without integrality requirements.

The present paper aims at paving some roads for optimal scenario reduction in chance constrained and mixed-integer two-stage stochastic programming models. Let $P$ be a discrete probability measure on $\mathbb{R}^{s}$ with support $\left\{\xi^{1}, \ldots, \xi^{N}\right\}$ and $P\left(\xi^{i}\right)=p_{i}>0$ for $i=1, \ldots, N$. We consider the problem of finding another discrete probability measure $Q$ on $\mathbb{R}^{s}$ which is supported on a subset of $\left\{\xi^{1}, \ldots, \xi^{N}\right\}$ and which deviates from $P$ as little as possible with respect to some discrepancy. We recall from $[10,9]$ that, for given Borel probability measures $P, Q$ on $\mathbb{R}^{s}$ and for a given system $\mathcal{B}$ of Borel subsets of $\mathbb{R}^{s}$, the $\mathcal{B}$-discrepancy between $P$ and $Q$ is defined as

$$
\alpha_{\mathcal{B}}(P, Q):=\sup _{B \in \mathcal{B}}|P(B)-Q(B)|
$$

Important examples are the systems $\mathcal{B}_{c l}$ of all closed subsets, $\mathcal{B}_{\text {conv }}$ of all closed, convex subsets, $\mathcal{B}_{\text {polyk }}$ of all polyhedra having at most $k$ vertices, $\mathcal{B}_{\text {rect }}$ of all closed, $s$-dimensional rectangles $\times_{i=1}^{s} I_{i}$ with $I_{i}, i=1, \ldots, s$, denoting a closed interval in $\mathbb{R}$, and $\mathcal{B}_{\text {cell }}$ of all closed cells (i.e., sets of the form $\xi+\mathbb{R}_{-}^{s}$ with $\xi \in \mathbb{R}^{s}$ ) of $\mathbb{R}^{s}$. Evidently, one has that

$$
\alpha_{\mathcal{B}_{\text {cell }}} \leq \alpha_{\mathcal{B}_{\text {rect }}} \leq \alpha_{\mathcal{B}_{\text {polyk }}} \leq \alpha_{\mathcal{B}_{\text {conv }}} \leq \alpha_{\mathcal{B}_{\text {cl }}}
$$

where for the inequality $\alpha_{\mathcal{B}_{\text {rect }}} \leq \alpha_{\mathcal{B}_{\text {polyk }}}$ one has to require that $k \geq 2^{s}$ (in order to ensure that $\left.\mathcal{B}_{\text {rect }} \subseteq \mathcal{B}_{\text {polyk }}\right)$. Any $\mathcal{B}$-discrepancy is a semimetric on the space of all probability measures on $\mathbb{R}^{s}$, i.e., it is non-negative, symmetric and satisfies the triangle equality. The discrepancy $\alpha_{\mathcal{B}_{\text {cell }}}$ (and, thus, all discrepancies in (1)) are metrics as, in addition, $\alpha_{\mathcal{B}_{\text {cell }}}(P, Q)=0$ implies $P=Q$. A sequence $\left(P_{n}\right)$ of probability measures converges to $P$ with respect to $\alpha_{\mathcal{B}}$ with $\mathcal{B} \subseteq \mathcal{B}_{c l}$ iff it converges weakly to $P$ and $P(\partial B)=0$ holds for each $B \in \mathcal{B}$ (with $\partial B$ denoting the boundary of $B$ ) [2]. We refer to the monograph [1] for further background on weak convergence of probability measures.

In the literature (cf. [16]), $\alpha_{\mathcal{B}_{\text {cell }}}$ is also called uniform or Kolmogorov metric as $\alpha_{\mathcal{B}_{\text {cell }}}(P, Q)$ is just the uniform distance of the probability distribution functions of $P$ and $Q$ on $\mathbb{R}^{s}$. The distance $\alpha_{\mathcal{B}_{\text {conv }}}$ is known as isotrope discrepancy [12] and $\alpha_{\mathcal{B}_{c l}}$ as total variation [16]. Consistently, the distance $\alpha_{\mathcal{B}_{\text {cell }}}\left(\alpha_{\mathcal{B}_{\text {rect }}}, \alpha_{\mathcal{B}_{\text {polyk }}}\right)$ will be called cell (rectangular, polyhedral) discrepancies. Some of these discrepancies have been extensively used for studying properties of uniformly distributed sequences in the $s$-dimensional unit cube $U^{s}=[0,1]^{s}[11]$ and, more recently, for developing Quasi-Monte Carlo methods [13]. Converse inequalities to (1), e.g., for the isotrope and rectangular discrepancies of probability measures $P$ and $Q$ on $\mathbb{R}^{s}$, were also derived $[9,11,12,14]$. For instance, the estimate

$$
\alpha_{\mathcal{B}_{\text {conv }}}(P, Q) \leq s\left(\frac{4 M s}{s-1}\right)^{\frac{s-1}{s}} \alpha_{\mathcal{B}_{\text {rect }}}(P, Q)^{\frac{1}{s}}
$$


holds if $P$ has a density (with respect to the Lebesgue measure on $\mathbb{R}^{s}$ ) which is bounded by $M$ [14]. In the context of quantitative stability of stochastic programs it is worth noting that the polyhedral, rectangular and cell discrepancies are of special importance for linear chance constrained and mixed-integer two-stage models [19, 22, 17, 18, 8].

Denoting by $\delta_{\xi}$ the Dirac-measure placing mass one at the point $\xi$, one may write the discrete measure $P$ introduced above as

$$
P=\sum_{i=1}^{N} p_{i} \delta_{\xi^{i}}
$$

where $\sum_{i=1}^{N} p_{i}=1$. Now, the reduction problem formulated above can be restated as the following optimization problem:

$$
\begin{aligned}
& \operatorname{minimize} \alpha_{\mathcal{B}}(P, Q)=\alpha_{\mathcal{B}}\left(\sum_{i=1}^{N} p_{i} \delta_{\xi^{i}}, \sum_{j=1}^{n} q_{j} \delta_{\eta^{j}}\right) \\
& \text { subject to }\left\{\eta^{1}, \ldots, \eta^{n}\right\} \subset\left\{\xi^{1}, \ldots, \xi^{N}\right\}, q_{j} \geq 0(j=1, \ldots, n), \sum_{j=1}^{n} q_{j}=1
\end{aligned}
$$

The variables to be optimally adjusted here are $\eta^{1}, \ldots, \eta^{n}$ and $q_{1}, \ldots, q_{n}$ and altogether they define the desired reduced discrete measure $Q$ via

$$
Q=\sum_{j=1}^{n} q_{j} \delta_{\eta^{j}}
$$

The optimization problem (3) may be decomposed into two subproblems: a combinatorial optimization problem for determining the scenario set $\eta=\left\{\eta^{1}, \ldots, \eta^{n}\right\}$ and a (linear) program for fixing $q=\left(q_{1}, \ldots, q_{n}\right)$. To describe this in more detail, we denote by $\alpha_{\mathcal{B}}(\eta, q)$ the $\mathcal{B}$-discrepancy between $P$ and $Q$ for fixed $P$, and by $S_{n}$ the standard simplex in $\mathbb{R}^{n}$, i.e.,

$$
\begin{aligned}
\alpha_{\mathcal{B}}(\eta, q) & :=\alpha_{\mathcal{B}}\left(\sum_{i=1}^{N} p_{i} \delta_{\xi^{i}}, \sum_{j=1}^{n} q_{j} \delta_{\eta^{j}}\right) \\
S_{n} & :=\left\{q \in \mathbb{R}^{n} \mid q_{j} \geq 0, j=1, \ldots, n, \sum_{j=1}^{n} q_{j}=1\right\}
\end{aligned}
$$

Now, the optimization problem (3) is of the form

$$
\min _{\eta}\left\{\inf _{q \in S_{n}} \alpha_{\mathcal{B}}(\eta, q) \mid \eta \subset\left\{\xi^{1}, \ldots, \xi^{N}\right\}, \# \eta=n\right\}
$$

where $\inf _{q \in S_{n}} \alpha_{\mathcal{B}}(\eta, q)$ refers to the infimum of the inner optimization model

$$
\min \left\{\alpha_{\mathcal{B}}(\eta, q) \mid q \in S_{n}\right\}
$$

for fixed scenario set $\eta$. While (5) represents a specific clustering problem, a so-called $k$-median problem of combinatorial optimization, the problem (6) will turn out as a linear program. Both problems will be further discussed in Section 3. In Section 2, we derive upper and lower bounds of the optimal value of (3) and discuss some particular cases, which allow for an explicit solution. In Section 4 we provide some preliminary numerical experience for optimal scenario reduction with respect to the cell discrepancy (or Kolmogorov distance). 


\section{Bounds and specific solutions}

In this section, we shall derive a specific solution for problem (3) in the case of the closed set discrepancy $\alpha_{\mathcal{B}}=\alpha_{\mathcal{B}_{c l}}$ as well as universal bounds for the optimal value of (3) in case of general discrepancies. By 'universal' we mean a bound that just depends on the probabilities $p_{i}$ of the original discrete measure $P$ but not on its support. In particular, these bounds do not depend on the geometry of the support or the space dimension $s$. Hence, in contrast to the exact solution of (3), these bounds are very easy to compute for a quite general class of discrepancies.

\subsection{Ordered solution and upper bound}

Intuitively, approximating the original discrete measure $P$ by some other measure $Q$ which is supported by a subset of the support of $P$, requires well to approximate those supporting points of $P$ having large probability. In this section, we assume, without loss of generality, that $p_{1} \geq \cdots \geq p_{N}$. Then, a naive idea for solving (3) would be to put in the definition (4) of $Q$ :

$$
\eta^{j}:=\xi^{j} \quad(j=1, \ldots, n) ; \quad q_{j}:=p_{j} \quad(j=1, \ldots, n-1) ; \quad q_{n}:=\sum_{i=n}^{N} p_{i} .
$$

This means that $Q$ selects its support as the atoms of $P$ having largest probability and, that the assignment of probabilities is adopted from the original measure except at the last atom, where the new probability is modified to make all $q_{j}$ sum up to one. Evidently, this simple approximating probability measure $Q$, which from now on shall be called the ordered solution, is feasible in (3). It has the interesting feature, that it realizes a universal (with respect to any discrepancy), easy to calculate upper bound in (3) which is actually sharp in case of the closed set discrepancy.

Proposition 2.1 As before, we assume, without loss of generality, that $p_{1} \geq \cdots \geq p_{N}$. Denote by $\Delta_{\mathcal{B}}$ the optimal value of (3), where $\mathcal{B}$ is any system of Borel measurable subsets of $\mathbb{R}^{s}$. Then, one has that

$$
\begin{aligned}
& \text { 1. } \Delta_{\mathcal{B}} \leq \sum_{i=n+1}^{N} p_{i} . \\
& \text { 2. } \Delta_{\mathcal{B}_{c l}}=\sum_{i=n+1}^{N} p_{i} \text {. }
\end{aligned}
$$

Proof. Define $Q$ in (4) as the ordered solution according to (7). Let $B \in \mathcal{B}$ be arbitrary and put

$$
J:=\left\{j \in\{1, \ldots, n\} \mid \xi^{j} \in B\right\} .
$$


Then, by (7),

$$
\begin{aligned}
& |P(B)-Q(B)| \\
= & \left|\sum_{i=1}^{N} p_{i} \delta_{\xi^{i}}(B)-\sum_{j=1}^{n} q_{j} \delta_{\eta^{j}}(B)\right| \\
= & \mid \sum_{i \in J \backslash\{n\}} p_{i} \delta_{\xi^{i}}(B)+p_{n} \delta_{\xi^{n}}(B)+\sum_{i=n+1}^{N} p_{i} \delta_{\xi^{i}}(B) \\
& -\sum_{j \in J \backslash\{n\}} q_{j} \delta_{\xi^{j}}(B)-q_{n} \delta_{\xi^{n}}(B) \mid \\
= & \left|\sum_{i \in J \backslash\{n\}} p_{i}+p_{n} \delta_{\xi^{n}}(B)+\sum_{i=n+1}^{N} p_{i} \delta_{\xi^{i}}(B)-\sum_{j \in J \backslash\{n\}} q_{j}-q_{n} \delta_{\xi^{n}}(B)\right| \\
= & \left|p_{n} \delta_{\xi^{n}}(B)-q_{n} \delta_{\xi^{n}}(B)+\sum_{i=n+1}^{N} p_{i} \delta_{\xi^{i}}(B)\right| \\
= & \left\{\begin{array}{ll}
\left|\sum_{i=n+1}^{N} p_{i} \delta_{\xi^{i}}(B)-\sum_{i=n+1}^{N} p_{i}\right| & \text { if } n \in J \\
\sum_{i=n+1}^{N} p_{i} \delta_{\xi^{i}}(B) & \text { if } n \notin J
\end{array} .\right.
\end{aligned}
$$

Due to

$$
0 \leq \sum_{i=n+1}^{N} p_{i} \delta_{\xi^{i}}(B) \leq \sum_{i=n+1}^{N} p_{i}
$$

one arrives at

$$
|P(B)-Q(B)| \leq \sum_{i=n+1}^{N} p_{i}
$$

Since $B \in \mathcal{B}$ was arbitrary, there follows assertion 1 .:

$$
\Delta_{\mathcal{B}} \leq \alpha_{\mathcal{B}}(P, Q)=\sup _{B \in \mathcal{B}}|P(B)-Q(B)| \leq \sum_{i=n+1}^{N} p_{i}
$$

Concerning assertion 2., let $Q$ in (4) be any discrete measure which is feasible in problem (3) with respect to the special discrepancy distance $\alpha_{\mathcal{B}}=\alpha_{\mathcal{B}_{c l}}$. Feasibility of $Q$ in (3) implies that $\left\{\eta^{1}, \ldots, \eta^{n}\right\} \subseteq\left\{\xi^{1}, \ldots, \xi^{N}\right\}$. Therefore, $\eta^{j}=\xi^{i_{j}}$ for certain selections $i_{j} \in$ $\{1, \ldots, N\}$ and $j=1, \ldots, n$. Since $B:=\left\{\xi^{1}, \ldots, \xi^{N}\right\} \backslash\left\{\eta^{1}, \ldots, \eta^{n}\right\}$ is a closed set, one derives that $B \in \mathcal{B}_{c l}$ and thus:

$$
\begin{aligned}
\alpha_{\mathcal{B}_{c l}}(P, Q) & \geq|P(B)-Q(B)| \\
& =\left|\sum_{i \in\left\{i_{1}, \ldots, i_{n}\right\}} p_{i} \delta_{\xi^{i}}(B)+\sum_{i \notin\left\{i_{1}, \ldots, i_{n}\right\}} p_{i} \delta_{\xi^{i}}(B)-\sum_{j=1}^{n} q_{j} \delta_{\eta^{j}}(B)\right| \\
& =\sum_{i \notin\left\{i_{1}, \ldots, i_{n}\right\}} p_{i} \geq \sum_{i=n+1}^{N} p_{i},
\end{aligned}
$$

where in the last inequality the assumed decreasing order of the $p_{i}$ was exploited. As $Q$ was supposed to be arbitrary feasible in (3), one gets that

$$
\Delta_{\mathcal{B}_{c l}}=\inf \left\{\alpha_{\mathcal{B}_{c l}}(P, Q) \mid Q \text { feasible in }(3)\right\} \geq \sum_{i=n+1}^{N} p_{i}
$$

Taking into account the reverse inequality, already proved in assertion 1 . for an arbitrary discrepancy, there follows assertion 2 . 
Corollary 2.1 The ordered solution defined in (7) is a (nonunique) optimal solution of problem (3) for the closed set discrepancy $\alpha_{\mathcal{B}_{c l}}$.

Proof. In the first part of the proof of Proposition 2.1, we have shown that, for any discrepancy $\alpha_{\mathcal{B}}$, the ordered solution realizes an objective value in (3) which is not larger than $\sum_{i=n+1}^{N} p_{i}$. On the other hand, the same value is optimal in (3) for the closed set discrepancy $\alpha_{\mathcal{B}_{c l}}$ (see assertion 2. in Prop. 2.1).

The last corollary shows, that in case of the closed set discrepancy, an explicit solution of problem (3) can be found without any computational effort. The same does not hold true for the weaker discrepancies mentioned in the introduction. Nevertheless, for those other discrepancies too, one may benefit from the upper bound information for the optimal value in (3) provided by the first statement in Proposition 2.1. For instance, from the (ordered) values of the original probabilities $p_{i}$, one can directly read off the number of atoms $n<N$ required for the approximating measure $Q$, in order to make the approximation error $\alpha_{\mathcal{B}}(P, Q)$ not exceed a prescribed tolerance $\varepsilon>0$. In the special case of $p_{i}=N^{-1}(i=$ $1, \ldots, N)$, one derives the condition

$$
\frac{n}{N} \geq 1-\varepsilon
$$

For instance, a tolerance of $10 \%(\varepsilon=0.1)$ can be satisfied then, if $n$ is at least $90 \%$ of $N$. Of course, such linear relation between tolerance and size of distribution is not very satisfactory. Indeed, the second assertion of Proposition 2.1 tells us, that, in the assumed equi-distributed case, one actually observes this undesirable linear relation for the closed set discrepancy. Consequently, there is some hope, that a better behaviour can be observed for the weaker discrepancies, which are more appropriate for the stability of chance constrained and mixed-integer stochastic programs (cf. Section 1). This, however, comes at the price that a simple solution of (3) is no longer available and, actually, cannot even be obtained computationally for relevant problem sizes in an exact sense.

The following example complements Corollary 2.1 by showing that the ordered solution need not be optimal for a discrepancy different from $\alpha_{\mathcal{B}_{c l}}$ :

Example 2.1 Define the original measure $P$ on $\mathbb{R}$ by

$$
\xi^{1}:=1, \xi^{2}:=3, \xi^{3}:=2, \xi^{4}:=4 ; \quad p_{1}:=p_{2}:=0.4 ; \quad p_{3}:=p_{4}:=0.1 .
$$

We are looking for the optimally reduced measure $Q$ in problem (3) which selects $n:=2$ atoms from the original measure. As far as this is done with respect to the closed set discrepancy $\alpha_{\mathcal{B}_{c l}}$, Corollary 2.1 guarantees that the ordered solution $Q$ defined by

$$
\eta^{1}:=1, \eta^{2}:=3, q_{1}:=0.4, q_{2}:=0.6
$$

is optimal and, by Proposition 2.1, realizes the minimal discrepancy $\Delta_{\mathcal{B}_{c l}}=p_{3}+p_{4}=0.2$. For the convex set discrepancy $\alpha_{\mathcal{B}_{\text {conv }}}$ (see introduction), this ordered solution realizes the same value $\alpha_{\mathcal{B}_{\text {conv }}}(P, Q)=0.2$. However, considering the reduced measure $Q^{*}$ defined by

$$
\eta^{1}:=1, \eta^{2}:=3, q_{1}:=0.5, q_{2}:=0.5,
$$


it follows that $\alpha_{\mathcal{B}_{\text {conv }}}\left(P, Q^{*}\right)=0.1$. Consequently, the ordered solution is not optimal in (3) with respect to $\alpha_{\mathcal{B}_{\text {conv }}}$. At the same time, this is an example for a strict inequality in statement 1. of Proposition 2.1.

\subsection{Lower bound}

In this section, we want to find a universal lower bound for the optimal value of problem (3). For this purpose, we will access on the following property.

Definition 2.1 We call a system $\mathcal{B}$ of Borel subsets of $\mathbb{R}^{s}$ isolating if for any finite subset $\left\{x^{1}, \ldots, x^{p}\right\} \subseteq \mathbb{R}^{s}$ there exist sets $B^{i} \in \mathcal{B}$ for $i=1, \ldots, p$ with

$$
B^{i} \cap\left\{x^{1}, \ldots, x^{p}\right\}=\left\{x^{i}\right\} \quad(i=1, \ldots, p) .
$$

Clearly, the systems $\mathcal{B}_{\text {rect }}, \mathcal{B}_{\text {conv }}, \mathcal{B}_{c l}$ and $\mathcal{B}_{\text {polyk }}$ (for $k \geq 2^{s}$ ) mentioned in the introduction are isolating, whereas $\mathcal{B}_{\text {cell }}$, for instance, is not.

Theorem 2.1 Let $\mathcal{B}$ be an isolating system of Borel subsets of $\mathbb{R}^{s}$. In (3), let $n<N$. Then, assuming as before that the $p_{i}$ are decreasingly ordered, the optimal value $\Delta_{\mathcal{B}}$ in problem (3) has the lower bound

$$
\Delta_{\mathcal{B}} \geq \max \left\{p_{n+1}, n^{-1} \sum_{i=n+1}^{N} p_{i}\right\} .
$$

Proof. Each measure $Q$ defined by (4) which is feasible in problem (3) induces an injective selection mapping $\sigma:\{1, \ldots, n\} \rightarrow\{1, \ldots, N\}$ with

$$
\eta^{i}=\xi^{\sigma(i)} \quad(i=1, \ldots, n) .
$$

Applying Definition 2.1 to the support $\left\{\xi^{1}, \ldots, \xi^{N}\right\}$ of the original measure $P$, we derive the existence of sets $B^{i} \in \mathcal{B}$ for $i=1, \ldots, N$ such that

$$
B^{i} \cap\left\{\xi^{1}, \ldots, \xi^{N}\right\}=\left\{\xi^{i}\right\} \quad(i=1, \ldots, N) .
$$

Then,

$$
\begin{aligned}
\left|P\left(B^{\sigma(i)}\right)-Q\left(B^{\sigma(i)}\right)\right| & =\left|P\left(\left\{\xi^{\sigma(i)}\right\}\right)-Q\left(\left\{\eta^{i}\right\}\right)\right|=\left|p_{\sigma(i)}-q_{i}\right| \quad(i=1, \ldots, n) \\
\left|P\left(B^{i}\right)-Q\left(B^{i}\right)\right| & =p_{i} \quad\left(i \in C_{\sigma}\right),
\end{aligned}
$$

where $C_{\sigma}:=\{1, \ldots, N\} \backslash\{\sigma(1), \ldots, \sigma(n)\}$. It follows for the discrepancy that

$$
\alpha_{\mathcal{B}}(P, Q) \geq \max _{i=1, \ldots, N}\left|P\left(B^{i}\right)-Q\left(B^{i}\right)\right|=\max \left\{\max _{i \in C_{\sigma}} p_{i}, \max _{i=1, \ldots, n}\left|p_{\sigma(i)}-q_{i}\right|\right\} .
$$

Note, that the variation of $Q$ among the feasible measures in (3) amounts to variation of the selection mapping $\sigma$ and to variation of coefficients $q_{i} \geq 0$ subject to the constraints $\sum_{i=1}^{n} q_{i}=1$. This allows to write

$$
\begin{aligned}
\Delta_{\mathcal{B}} & =\inf \left\{\alpha_{\mathcal{B}}(P, Q) \mid Q \text { feasible in }(3)\right\} \\
& \geq \inf \{\varphi(\sigma) \mid \sigma:\{1, \ldots, n\} \rightarrow\{1, \ldots, N\} \text { injective }\},
\end{aligned}
$$


where

$$
\begin{aligned}
& \varphi(\sigma):=\max \left\{\max _{i \in C_{\sigma}} p_{i}, \psi(\sigma)\right\} \\
& \psi(\sigma):=\inf \left\{\max _{i=1, \ldots, n}\left|p_{\sigma(i)}-q_{i}\right| \mid q_{i} \geq 0 \quad(i=1, \ldots, n), \quad \sum_{i=1}^{n} q_{i}=1\right\} .
\end{aligned}
$$

Next, we want to develop the expression for $\psi(\sigma)$. Since $p_{i}>0$ for $i=1, \ldots, N$ and $n<N$, by assumption, it follows that

$$
\gamma:=\sum_{i=1}^{n} p_{\sigma(i)}<1
$$

Note that the infimum in the definition of $\psi(\sigma)$ is always realized as a minimum. We claim that $\hat{q} \in \mathbb{R}^{n}$ defined by

$$
\hat{q}_{i}=p_{\sigma(i)}+n^{-1}(1-\gamma) \quad(i=1, \ldots, n)
$$

provides this minimum. We have $\hat{q}_{i} \geq 0$ for $i=1, \ldots, n$ due to $\gamma<1$ and $\sum_{i=1}^{n} \hat{q}_{i}=1$, hence $\hat{q}$ is feasible in the definition of $\psi(\sigma)$. Now, let $q^{\prime} \in \mathbb{R}^{n}$ be any other feasible $n$-tupel. Then, by

$$
\sum_{i=1}^{n} q_{i}^{\prime}=\sum_{i=1}^{n} \hat{q}_{i}=1
$$

it is excluded that $q_{i}^{\prime}<\hat{q}_{i}$ holds true for all $i=1, \ldots, n$. Consequently, there exists some $k \in\{1, \ldots, n\}$ with $q_{k}^{\prime} \geq \hat{q}_{k}$. From the relation $\hat{q}_{k} \geq p_{\sigma(k)}$ (see (9)), one derives that $\left|p_{\sigma(k)}-q_{k}^{\prime}\right| \geq\left|p_{\sigma(k)}-\hat{q}_{k}\right|$. Thus,

$$
\max _{i=1, \ldots, n}\left|p_{\sigma(i)}-q_{i}^{\prime}\right| \geq\left|p_{\sigma(k)}-\hat{q}_{k}\right|=n^{-1}(1-\gamma)=\max _{i=1, \ldots, n}\left|p_{\sigma(i)}-\hat{q}_{i}\right| .
$$

This shows that indeed $\hat{q}$ realizes the infimum in the definition of $\psi(\sigma)$ and so, by (9) and by definition of $C_{\sigma}$, one gets that

$$
\psi(\sigma)=n^{-1}(1-\gamma)=n^{-1}\left(1-\sum_{i=1}^{n} p_{\sigma(i)}\right)=n^{-1} \sum_{i \in C_{\sigma}} p_{i} .
$$

Now, we continue (8) as

$$
\Delta_{\mathcal{B}} \geq \inf \left\{\max \left\{\max _{i \in C_{\sigma}} p_{i}, n^{-1} \sum_{i \in C_{\sigma}} p_{i}\right\} \mid \sigma:\{1, \ldots, n\} \rightarrow\{1, \ldots, N\} \text { injective }\right\} .
$$

Identifying the set of all selections as given in this relation with the system of all subsets of $\{1, \ldots, N\}$ having cardinality $n$, one obtains the reformulation

$$
\Delta_{\mathcal{B}} \geq \inf \left\{\max \left\{\max _{i \in A} p_{i}, n^{-1} \sum_{i \in A} p_{i}\right\} \mid A \subseteq\{1, \ldots, N\}, \# A=N-n\right\} .
$$

As the $p_{i}$ are decreasingly ordered, both expressions

$$
\max _{i \in A} p_{i} \text { and } n^{-1} \sum_{i \in A} p_{i}
$$


are simultaneously minimized by the set $A^{*}:=\{n+1, \ldots, N\}$. Therefore,

$$
\Delta_{\mathcal{B}} \geq \max \left\{\max _{i \in\{n+1, \ldots, N\}} p_{i}, n^{-1} \sum_{i=n+1}^{N} p_{i}\right\} .
$$

Owing to $\max \left\{p_{i} \mid i \in\{n+1, \ldots, N\}\right\}=p_{n+1}$, the assertion of the theorem is proved.

Remark 2.1 The lower bound from Theorem 2.1 can be interpreted as follows. Consider an arbitrary reduced measure $Q$. Since $\mathcal{B}$ is isolating, the $\mathcal{B}$-discrepancy between $P$ and $Q$ is larger than the maximal difference of $P$ and $Q$ on a singleton. Over all common mass points of $P$ and $Q$, this maximum is at least $n^{-1} \sum_{i=n+1}^{N} p_{i}$, over all points without $Q$-mass it is not less than $p_{n+1}$.

Corollary 2.2 Under the assumptions of Theorem 2.1, the following holds true:

1. If $n \geq \frac{N}{2}$, then the lower bound in Theorem 2.1 reduces to $p_{n+1}$.

2. If $n=1$, then $\Delta_{\mathcal{B}}=1-p_{1}$ and the optimal solution of (3) is given by the measure $Q$ placing unit mass on the atom realizing maximum probability with respect to the original measure $P$.

If $n=N-1$, then $\Delta_{\mathcal{B}}=p_{N}$, and any measure $Q_{j}$ of the form

$$
Q_{j}=\sum_{i=1, i \neq j}^{N-1} p_{i} \delta_{\xi^{i}}+\left(p_{j}+p_{N}\right) \delta_{\xi^{j}} \quad j \in\{1, \ldots, N-1\}
$$

is an optimal solution of (3).

Proof. The decreasing order of the $p_{i}$ implies 1 . by Theorem 2.1 and the estimate

$$
n p_{n+1} \geq(N-n) p_{n+1} \geq \sum_{i=n+1}^{N} p_{i}
$$

which proves the first statement.

In both cases $n=1$ and $n=N-1$, Theorem 2.1 provides that $\Delta_{\mathcal{B}} \geq \sum_{i=n+1}^{N} p_{i}$. Now, the upper bound in Proposition 2.1 turns this inequality into an equality:

$$
\Delta_{\mathcal{B}}=\sum_{i=2}^{N} p_{i}= \begin{cases}1-p_{1} & \text { if } n=1 \\ p_{N} & \text { if } n=N-1\end{cases}
$$

From the proof of statement 1. in Proposition 2.1) we know that the ordered solution always realizes a discrepancy not larger than $\sum_{i=2}^{N} p_{i}$., where this last value was just recognized to be optimal for $n=1$ and $n=N-1$. Consequently, the ordered solution must be optimal in these cases. For $n=1$, the ordered solution places unit mass on the atom with highest probability in the original measure $P$. For $n=N-1$, the ordered solution corresponds to the measure $Q_{N-1}$. Since $\mathcal{B}$ is isolating, the measure $Q_{j}$ is optimal for any $j \in\{1, \ldots, N-1\}$.

Unfortunately, the results in Corollary 2.2 are lost for the cell discrepancy $\alpha_{\mathcal{B}_{\text {cell }}}$ as the next example shows.

Example 2.2 Consider the probability measure $P=\sum_{i=1}^{3} p_{i} \delta_{\xi^{i}}$ on $\mathbb{R}^{2}$ with ordered probabilities $p_{1} \geq p_{2} \geq p_{3}$ and $\xi^{1}=(1,1), \xi^{2}=(1,0.5), \xi^{3}=(2,2)$. For $n=1$ we obtain $\Delta_{\mathcal{B}_{\text {cell }}}=\alpha_{\mathcal{B}_{\text {cell }}}\left(P, \delta_{\xi^{1}}\right)=p_{2}$, which contradicts to the results of Corollary 2.2. 


\section{Solution techniques}

As mentioned in Section 1 problem (3) can be tackled by a bilevel approach: in an outer iteration, the support selection is carried out by solving the combinatorial optimization problem (5), whereas in an inner iteration optimal probabilities $q_{j}$ are determined conditional to the fixed support by solving (6). Since problem (5) is known to be NP-hard, we resort to applying heuristic approaches. For two-stage models certain forward selection and backward reduction techniques are developed in [4]. In the context of the present paper, their analogues represent recursive extensions of the cases $n=1$ and $n=N-1$ in Corollary 2.2. The forward and backward algorithms determine index subsets $J^{[n]}$ and $J^{[N-n]}$, respectively, of $\{1, \ldots, N\}$. Both index sets are of cardinality $n$ and the corresponding scenario sets form the support of the reduced probability measure $Q$.

Algorithm 3.1 (Forward selection)

$$
\begin{aligned}
\text { Step [0]: } & J^{[0]}:=\emptyset . \\
\text { Step [i]: } & l_{i} \in \operatorname{argmin}_{l \notin J}[i-1] \inf _{q \in S_{i}} \alpha_{\mathcal{B}}\left(\left\{\xi^{l_{1}}, \ldots, \xi^{l_{i-1}}, \xi^{l}\right\}, q\right), \\
& J^{[i]}:=J^{[i-1]} \cup\left\{l_{i}\right\} . \\
\text { Step [n+1]: } & \text { Minimize } \alpha_{\mathcal{B}}\left(\left\{\xi^{l_{1}}, \ldots, \xi^{l_{n}}\right\}, q\right) \text { subject to } q \in S_{n} .
\end{aligned}
$$

Algorithm 3.2 (Backward reduction)

$$
\begin{aligned}
\text { Step [0]: } & J^{[0]}:=\{1, \ldots, N\} . \\
\text { Step [i]: } & u_{i} \in \operatorname{argmin}_{u \in J}[i-1] \inf _{q \in S_{N-i}} \alpha_{\mathcal{B}}\left(\left\{\xi^{j} \mid j \in J^{[i-1]} \backslash\{u\}\right\}, q\right), \\
& J^{[i]:=J^{[i-1]} \backslash\left\{u_{i}\right\} .} \\
\text { Step [N-n+1]: } & \text { Minimize } \alpha_{\mathcal{B}}\left(\left\{\xi^{j} \mid j \in J^{[N-n]}\right\}, q\right) \text { subject to } q \in S_{n} .
\end{aligned}
$$

Note that, at each Step [i], $i=1, \ldots, n$, one has to solve $N-i$ linear programs, which are of dimension $i$ and $N-i$ in Algorithms 3.1 and 3.2, respectively. Hence, forward selection seems to be preferable in most relevant cases. Although both algorithms do not lead to optimality in (3) in general, the performance evaluation of their implemented analogues for transportation distances in $[4,6]$ is encouraging.

In the following, we want to consider the inner iteration problem of optimizing the probability distribution conditional to a fixed support. Without loss of generality, we may assume that $\left\{\eta^{1}, \ldots, \eta^{n}\right\}=\left\{\xi^{1}, \ldots, \xi^{n}\right\}$. Of course, we may no longer maintain then the assumption of ordered probabilities $p_{i}$ from the previous section without restricting the generality. Anyway, ordered probabilities $p_{i}$ are no longer relevant in the sequel. Then, problem (6) is of the form:

$$
\operatorname{minimize} \alpha_{\mathcal{B}}\left(\left\{\xi^{1}, \ldots, \xi^{n}\right\}, q\right)=\alpha_{\mathcal{B}}\left(\sum_{i=1}^{N} p_{i} \delta_{\xi^{i}}, \sum_{j=1}^{n} q_{j} \delta_{\xi^{j}}\right) \text { subject to } q \in S_{n} .
$$




\subsection{Formulation as a linear optimization problem}

In this section, we are going to reformulate (10) as a linear optimization problem. For $B \in \mathcal{B}$, define a 'critical index set' $I(B) \subseteq\{1, \ldots, N\}$ by the relation

$$
B \cap\left\{\xi^{1}, \ldots, \xi^{N}\right\}=\left\{\xi^{i} \mid i \in I(B)\right\} .
$$

Then,

$$
|P(B)-Q(B)|=\left|\sum_{i \in I(B)} p_{i}-\sum_{j \in I(B) \cap\{1, \ldots, n\}} q_{j}\right|
$$

Obviously, this value does not depend on the concrete structure of the set $B$ but is uniquely determined by the index set $I(B)$. That is why, for calculating the discrepancy $\alpha_{\mathcal{B}}(P, Q)$, it suffices to know all (finitely many) critical index sets which may occur when $B$ varies in $\mathcal{B}$. We define the system of critical index sets as

$$
\mathcal{I}_{\mathcal{B}}:=\{I \subseteq\{1, \ldots, N\} \mid \exists B \in \mathcal{B}: I=I(B)\} .
$$

For the closed set discrepancy, for instance, one has $\mathcal{I}_{\mathcal{B}_{c l}}=2^{\{1, \ldots, N\}}$, because for an arbitrary subset $I \subseteq\{1, \ldots, N\}$ and an arbitrary $\xi^{i}$ with $i \in I$, one may find a small closed ball $B_{i} \supseteq\left\{\xi^{i}\right\}$, such that $\xi_{j} \notin B_{i}$ for all $j \in\{1, \ldots, N\} \backslash\{i\}$. Consequently, $B:=\cup_{i \in I} B_{i} \in \mathcal{B}_{c l}$ satisfies $B \cap\left\{\xi^{1}, \ldots, \xi^{N}\right\}=\left\{\xi^{i} \mid i \in I\right\}$, so $I(B)=I$. For the other systems $\mathcal{B}$ considered in the introduction, all one usually gets, is the strict inclusion $\mathcal{I}_{\mathcal{B}} \subset 2^{\{1, \ldots, N\}}$.

As soon as for some concrete $\mathcal{B}$ the system $\mathcal{I}_{\mathcal{B}}$ of critical index sets is known, the discrepancy between $P$ and $Q$ may be calculated according to (11) by using the formula

$$
\alpha_{\mathcal{B}}(P, Q)=\max _{I \in \mathcal{I}_{\mathcal{B}}}\left|\sum_{i \in I} p_{i}-\sum_{j \in I \cap\{1, \ldots, n\}} q_{j}\right| .
$$

We recall the well-kown fact that minimizing a function $|f(x)|$ in terms of the variable $x$ is equivalent to minimizing the function $t$ subject to the constraints $f(x) \leq t$ and $-f(x) \leq t$ in terms of the variables $(x, t)$. This allows to solve (10) by means of the following linear optimization problem:

$$
\begin{aligned}
& \operatorname{minimize} t \quad \text { subject to } q \in S_{n} \\
& \left.\begin{array}{c}
-\sum_{j \in I \cap\{1, \ldots, n\}} q_{j} \leq t-\sum_{i \in I} p_{i} \\
\sum_{j \in I \cap\{1, \ldots, n\}} q_{j} \leq t+\sum_{i \in I} p_{i}
\end{array}\right\} I \in \mathcal{I}_{\mathcal{B}} .
\end{aligned}
$$

The variables to be optimized here, are $t$ and the $q_{j}$. If $\left(q^{*}, t^{*}\right)$ is an optimal solution of (12), then $q^{*}$ is an optimal solution of the original problem (10), whereas $t^{*}$ indicates the optimal value attained by $q^{*}$ in $(12)$, i.e., $t^{*}$ provides the minimal discrepancy $\alpha_{\mathcal{B}}(P, Q)$ between the original measure $P$ and any measure $Q$ whose support coincides with the first $n$ points of the support of $P$. 
Unfortunately, the size of (12) is too large to be useful, in general. Indeed, since $\mathcal{I}_{\mathcal{B}_{c l}}=$ $2^{\{1, \ldots, N\}}$, as observed above, the number of constraints in (12) amounts to $2^{N+1}+n+1$. On the other hand, one recognizes from (12), that many inequalities are just copies of themselves as far as the involved coefficients $q_{j}$ are concerned, because many different index sets $I \in \mathcal{I}_{\mathcal{B}}$ may lead to the same intersection $I \cap\{1, \ldots, n\}$. The only term which varies then for those sets $I$, is the right-hand side of the inequalities in (12). Consequently, one may pass to the minimum of these right-hand sides corresponding to one and the same intersection $I \cap\{1, \ldots, n\}$ which will drastically reduce the number of inequalities. In order to do so formally correct, we introduce a reduced system of critical index sets as

$$
\mathcal{I}_{\mathcal{B}}^{*}:=\left\{I \cap\{1, \ldots, n\} \mid I \in \mathcal{I}_{\mathcal{B}}\right\} .
$$

Each member $J \in \mathcal{I}_{\mathcal{B}}^{*}$ of the reduced system generates a set $\varphi(J)$ of members in the original system $\mathcal{I}_{\mathcal{B}}$ all of which share the same intersection with $\{1, \ldots, n\}$ :

$$
\varphi(J):=\left\{I \in \mathcal{I}_{\mathcal{B}} \mid J=I \cap\{1, \ldots, n\}\right\} \quad\left(J \in \mathcal{I}_{\mathcal{B}}^{*}\right) .
$$

Now, introducing the quantities

$$
\gamma^{J}:=\max _{I \in \varphi(J)} \sum_{i \in I} p_{i} \quad \text { and } \quad \gamma_{J}:=\min _{I \in \varphi(J)} \sum_{i \in I} p_{i} \quad\left(J \in \mathcal{I}_{\mathcal{B}}^{*}\right)
$$

(12) may be rewritten as

$$
\begin{aligned}
& \text { minimize } t \text { subject to } q \in S_{n} \text {, } \\
& \left.\begin{array}{ll}
-\sum_{j \in J} q_{j} & \leq t-\gamma^{J} \\
\sum_{j \in J} q_{j} & \leq t+\gamma_{J}
\end{array}\right\} J \in \mathcal{I}_{\mathcal{B}}^{*}
\end{aligned}
$$

This corresponds indeed to passing to the minimum on the right-hand sides of the inequalities in (12). Since $\mathcal{I}_{\mathcal{B}}^{*}$ is a subset of $\{1, \ldots, n\}$, the number of inequalities in (15) is not larger than $2^{n}+n+1$. Having in mind that often $n \ll N$, this results in a drastic reduction of size in the linear optimization problem (12).

The linear constraints for each $J \in \mathcal{I}_{\mathcal{B}}^{*}$ imply that every feasible $t$ of problem (15) satisfies $t \geq \frac{1}{2}\left(\gamma^{J}-\gamma_{J}\right)$ and, thus, one obtains the lower bound

$$
\frac{1}{2} \max _{J \in \mathcal{I}_{\mathcal{B}}^{*}}\left(\gamma^{J}-\gamma_{J}\right) \leq \inf _{q \in S_{n}} \alpha_{\mathcal{B}}\left(\left\{\xi^{1}, \ldots, \xi^{n}\right\}, q\right) .
$$

Hence, if $q^{*} \in S_{n}$ satisfies the conditions

$$
\gamma^{J}-\frac{1}{2} \max _{J \in \mathcal{I}_{\mathcal{B}}^{*}}\left(\gamma^{J}-\gamma_{J}\right) \leq \sum_{j \in J} q_{j}^{*} \leq \frac{1}{2} \max _{J \in \mathcal{I}_{\mathcal{B}}^{*}}\left(\gamma^{J}-\gamma_{J}\right)+\gamma_{J} \quad\left(J \in \mathcal{I}_{\mathcal{B}}^{*}\right),
$$

implying, in particular,

$$
\sum_{j \in J_{*}} q_{j}^{*}=\frac{1}{2}\left(\gamma^{J_{*}}+\gamma_{J_{*}}\right) \quad\left(J_{*} \in \operatorname{argmax}_{J \in \mathcal{I}_{\mathcal{B}}^{*}}\left(\gamma^{J}-\gamma_{J}\right)\right),
$$

then the pair $\left(q^{*}, \frac{1}{2} \max _{J \in \mathcal{I}_{\mathcal{B}}^{*}}\left(\gamma^{J}-\gamma_{J}\right)\right)$ is an optimal solution of $(15)$. 


\subsection{The special case of the cell discrepancy $\alpha_{\mathcal{B}_{\text {cell }}}$}

The main challenge in the solution of $(15)$ is not the solution of the linear program itself but the computational determination of the reduced critical index set $\mathcal{I}_{\mathcal{B}}^{*}$ and of the coefficients $\gamma^{J}$ and $\gamma_{J}$ introduced in (14). As these strongly depend on the geometric structure of the chosen system $\mathcal{B}$ of Borel subsets, there is no general procedure available for this determination. In this section, an algorithmic approach for dealing with the special case of the cell discrepancy $\alpha_{\mathcal{B}_{\text {cell }}}$ shall be presented. The same methodology can be carried over to the rectangle discrepancy $\alpha_{\mathcal{B}_{\text {rect }}}$ though with higher computational effort. The more general discrepancies $\alpha_{\mathcal{B}_{\text {polyk }}}$ and $\alpha_{\mathcal{B}_{\text {conv }}}$ of polyhedra and closed convex sets, would require more sophisticated approaches which are outside the scope of this paper.

Recall that the sytem of cells is defined by

$$
\mathcal{B}_{\text {cell }}=\left\{z+\mathbb{R}_{-}^{s} \mid z \in \mathbb{R}^{s}\right\}
$$

where $\mathbb{R}_{-}^{s}=\left\{x \in \mathbb{R}^{s} \mid x_{j} \leq 0(j=1, \ldots, s)\right\}$ is the negative orthant of $\mathbb{R}^{s}$. For the purpose of abbreviation, we put $[z]:=z+\mathbb{R}_{-}^{s}$ for $z \in \mathbb{R}^{s}$.

Since the support $\left\{\xi^{1}, \ldots, \xi^{N}\right\}$ of the measure $P$ is finite, it is contained in an open rectangle $(c, d)$ for some $c, d \in \mathbb{R}^{s}$. We introduce an artificial point set $\left\{r^{1}, \ldots, r^{s}\right\}$ in order to control the boundary of the support, where $r^{j}$ is defined by

$$
r_{j}^{j}:=d_{j} \text { and } r_{k}^{j}:=c_{k} \text { if } k \neq j .
$$

Recall that the support of any feasible reduced measure $Q$ in (10) is given by the set $\left\{\xi^{1}, \ldots, \xi^{n}\right\}$ of first $n$ atoms of the support of $P$. We will show that it is sufficient to consider those cells, which are bounded in every direction by an element of

$$
R:=\left\{\xi^{1}, \ldots, \xi^{n}\right\} \cup\left\{r^{1}, \ldots, r^{s}\right\}
$$

in the following sense.

Definition 3.1 A cell $[z]$ is called supporting, if there exists a subset $\left\{x^{1}, \ldots, x^{s}\right\} \subseteq R$ such that $\left\{x^{1}, \ldots, x^{s}\right\} \subseteq[z]$ and $x_{j}^{j}=z_{j}$ for $j=1, \ldots, s$.

Indeed, we can restrict ourselves on supporting cells as shown by the following proposition, which relies on the fact that every cell can be enlarged until it is a supporting one. As the proof of this proposition is rather technical, it is given in the appendix.

Proposition 3.1 For any $J \in \mathcal{I}_{\mathcal{B}_{\text {cell }}}^{*}$, there exists a supporting cell $[z]$ such that $\gamma^{J}=$ $P($ int $[z])$ and

$$
\cup_{j \in J}\left\{\xi^{j}\right\}=\left\{\xi^{1}, \ldots, \xi^{n}\right\} \cap \operatorname{int}[z] .
$$

Before drawing some essential conclusions from Proposition 3.1, we mention the obvious fact that for any $z \in \mathbb{R}^{s}$ and any finite subset $F \subseteq \mathbb{R}^{s}$, there exists some $\varepsilon>0$, such that

$$
\left[z^{\varepsilon}\right] \cap F=\operatorname{int}[z] \cap F
$$

where $z^{\varepsilon}$ is defined by $z_{j}^{\varepsilon}:=z_{j}-\varepsilon$ for $j=1, \ldots, s$. 
Corollary 3.1 Define

$$
\mathcal{Z}:=\left\{z \in \mathbb{R}^{s} \mid[z] \text { is a supporting cell }\right\}
$$

Then,

$$
\begin{aligned}
\mathcal{I}_{\mathcal{B}_{\text {cell }}}^{*} & =\{J \subseteq\{1, \ldots, n\} \mid \exists z \in \mathcal{Z}:(17) \text { holds true }\} \\
\gamma^{J} & =\max \{P(\operatorname{int}[z]) \mid z \in \mathcal{Z},(17) \text { holds true }\} \quad \forall J \in \mathcal{I}_{\mathcal{B}_{\text {cell }}}^{*} .
\end{aligned}
$$

Proof. The inclusion ' $\subseteq$ ' in the first identity follows directly from the second statement of Proposition 3.1. Similarly, the inequality ' $\leq$ ' in the second identity follows directly from the first statement of Proposition 3.1. For the reverse direction of the first identity, let $z \in \mathcal{Z}$ be given such that (17) holds true for some $J \subseteq\{1, \ldots, n\}$. Now, we apply (18) to $F:=\left\{\xi^{1}, \ldots, \xi^{n}\right\}$ to derive the existence of some $z^{\varepsilon}$ such that

$$
\left\{\xi^{1}, \ldots, \xi^{n}\right\} \cap\left[z^{\varepsilon}\right]=\left\{\xi^{1}, \ldots, \xi^{n}\right\} \cap \operatorname{int}[z]=\cup_{j \in J}\left\{\xi^{j}\right\} .
$$

Since $\left[z^{\varepsilon}\right] \in \mathcal{B}_{\text {cell }}$, we observe upon recalling the definition of $I(B)$ for $B \in \mathcal{B}_{\text {cell }}$ in the beginning of section 3.1, that

$$
I\left(\left[z^{\varepsilon}\right]\right)=\left\{i \in\{1, \ldots, N\} \mid \xi^{i} \in\left[z^{\varepsilon}\right]\right\}=J \cup\left\{i \in\{n+1, \ldots, N\} \mid \xi^{i} \in\left[z^{\varepsilon}\right]\right\} .
$$

Therefore,

$$
I\left(\left[z^{\varepsilon}\right]\right) \cap\{1, \ldots, n\}=J \cap\{1, \ldots, n\}=J,
$$

which provides $J \in \mathcal{I}_{\mathcal{B}_{\text {cell }}}^{*}$ via the definition of $\mathcal{I}_{\mathcal{B}_{\text {cell }}}^{*}$. This shows the inclusion ' $\supseteq$ ' in the first identity. Concerning the reverse direction of the second identity, let $J \in \mathcal{I}_{\mathcal{B}_{\text {cell }}}^{*}$ and $z \in \mathcal{Z}$ be arbitrary, such that (17) holds true. Applying again (18), this time consecutively to $\left\{\xi^{1}, \ldots, \xi^{n}\right\}$ and to $\left\{\xi^{1}, \ldots, \xi^{N}\right\}$, one deduces the existence of some $z^{\varepsilon}$ such that (19) and

$$
\left\{\xi^{1}, \ldots, \xi^{N}\right\} \cap\left[z^{\varepsilon}\right]=\left\{\xi^{1}, \ldots, \xi^{N}\right\} \cap \operatorname{int}[z]
$$

hold true simultaneously. From (19) it follows (20) as in the lines above. Therefore, $I\left(\left[z^{\varepsilon}\right]\right) \in \varphi(J)($ see $(13))$ and

$$
\gamma^{J} \geq \sum_{i \in I\left(\left[z^{\varepsilon}\right]\right)} p_{i}=\sum_{\xi^{i} \in\left[z^{\varepsilon}\right]} P\left(\xi^{i}\right)=P\left(\left[z^{\varepsilon}\right]\right)=P(\operatorname{int}[z]),
$$

where the last equality relies on (21). Since $z \in \mathcal{Z}$ was chosen arbitrarily such that (17) holds true, this shows the inequality ' $\geq$ ' in the second identity.

Corollary 3.1 suggests that one can calculate the index family $\mathcal{I}_{\mathcal{B}_{\text {cell }}}^{*}$ as well as all upper coefficients $\gamma^{J}$ for $J \in \mathcal{I}_{\mathcal{B}_{\text {cell }}}^{*}$, as soon as one knows the set $\mathcal{Z}$ of supporting cells which is finite. Indeed, it follows from Definition 3.1, that each supporting cell is defined by an $s$-tupel $\left\{x^{1}, \ldots, x^{s}\right\}$. Consequently, one may extract the finite system of all $s$-tupels $\left\{x^{1}, \ldots, x^{s}\right\}$ out of the set $R=\left\{\xi^{1}, \ldots, \xi^{n}\right\} \cup\left\{r^{1}, \ldots, r^{s}\right\}$ and check for each of its members, whether this $s$-tupel defines a supporting cell. If so, this cell contributes to the calculation of $\mathcal{I}_{\mathcal{B}_{\text {cell }}}^{*}$ and of $\gamma^{J}$ for $J \in \mathcal{I}_{\mathcal{B}_{\text {cell }}}^{*}$. It remains to determine the lower coefficients $\gamma_{J}$ for $J \in \mathcal{I}_{\mathcal{B}_{\text {cell }}}^{*}$. 
Proposition 3.2 For all $J \in \mathcal{I}_{\mathcal{B}_{\text {cell }}^{*}}$, one has $\gamma_{J}=\sum_{i \in I} p_{i}$, where

$$
I:=\left\{i \in\{1, \ldots, N\} \mid \xi_{k}^{i} \leq \max _{j \in J} \xi_{k}^{j} \quad(k=1, \ldots, s)\right\} .
$$

Proof. Completely analogous to the derivation of (22) in the appendix, one obtains that

$$
\gamma_{J}=\min \left\{P([y]) \mid[y] \cap\left\{\xi^{1}, \ldots, \xi^{n}\right\}=\cup_{j \in J}\left\{\xi^{j}\right\}\right\} .
$$

Define $z \in \mathbb{R}^{s}$ by $z_{k}:=\max _{j \in J} \xi_{k}^{j}$ for $k=1, \ldots, s$. Then, $\xi^{j} \in[z]$ for all $j \in J$ and, thus,

$$
\cup_{j \in J}\left\{\xi^{j}\right\} \subseteq[z] \cap\left\{\xi^{1}, \ldots, \xi^{n}\right\}
$$

Assume that this inclusion is strict. Then, there is some $i \in\{1, \ldots, n\} \backslash J$ such that $\xi^{i} \in[z] . J \in \mathcal{I}_{\mathcal{B}_{\text {cell }}^{*}}$ means that there exists some $B \in \mathcal{B}_{\text {cell }}$ with $J=I(B) \cap\{1, \ldots, n\}$. Consequently, $\xi^{j} \in B$ for all $j \in J$, which entails that $[z] \subseteq B$, by construction of $z$. We derive that $\xi^{i} \in B$ and, hence, $i \in I(B)$. On the other hand, $i \in\{1, \ldots, n\} \backslash J$, which is a contradiction. It follows that

$$
\cup_{j \in J}\left\{\xi^{j}\right\}=[z] \cap\left\{\xi^{1}, \ldots, \xi^{n}\right\} .
$$

and, thus, $\gamma_{J} \leq P([z])$. On the other hand, if $y \in \mathbb{R}^{s}$ is arbitrary feasible in the definition of $\gamma_{J}$, then $\xi^{j} \in[y]$ for all $j \in J$, and so, $[z] \subseteq[y]$ again by construction of $z$. Now, $P([z]) \leq$ $P([y])$ which, upon passing to the minimum over all feasible $y$, provides $P([z]) \leq \gamma_{J}$. We may conclude that

$$
\gamma_{J}=P([z])=\sum_{\xi^{i} \in[z]} p_{i}
$$

which proves the assertion of the proposition.

\section{Algorithm and numerical results}

The results of the previous sections suggest the following algorithmic approach for the solution of problem (10) in the special case of the cell discrepancy $\alpha_{\mathcal{B}_{\text {cell }}}$, given the problem data $N, n, s,\left\{\xi^{1}, \ldots, \xi^{N}\right\}$ and $\left\{p_{1}, \ldots, p_{N}\right\}$ : 


\section{Algorithm 4.1}

Step [1]: $\quad$ To find an open rectangle $(c, d)$ such that $\left\{\xi^{1}, \ldots, \xi^{N}\right\} \subseteq(c, d)$, define $c_{j}:=\min _{i=1, \ldots, N} \xi_{j}^{i}-1, d_{j}:=\max _{i=1, \ldots, N} \xi_{j}^{i}+1 \quad(j=1, \ldots, s)$.

Calculate the set $R$ from Definition 3.1 with points $r^{j}$ defined in (16).

Put $\mathcal{I}_{\mathcal{B}}^{*}:=\{\emptyset\}, \mathcal{A}^{\prime}:=\{\emptyset\}$ and $\gamma^{J}:=0$ for all $J \subseteq\{1, \ldots, n\}$.

Let $\mathcal{A}:=\{A \subseteq\{1, \ldots, n+s\} \mid \# A=s\}$.

Step [2]: If $\mathcal{A}^{\prime}=\mathcal{A}$ then go to Step [7] else select $A=\left\{i_{1}, \ldots, i_{s}\right\} \in \mathcal{A} \backslash \mathcal{A}^{\prime}$.

Put $\mathcal{A}^{\prime}:=\mathcal{A}^{\prime} \cup\{A\}$ and

$x^{j}:=\left\{\begin{array}{ll}\xi^{i_{j}} & \text { if } i_{j} \leq n \\ r^{i_{j}-n} & \text { if } i_{j}>n\end{array} \quad(j=1, \ldots, s)\right.$.

Then, $x^{j} \in R$ for $j=1, \ldots, s$.

Step [3]: Define a 'potential' supporting cell $[z]$ for some $z \in \mathbb{R}^{s}$ by

$z_{k}:=\max _{j=1, \ldots, s} x_{k}^{j}(k=1, \ldots, s)$.

Check whether it is indeed a supporting cell according to Definition 3.1.

If not so, then go to Step [2].

Step [4]: Define $J$ by the relation (17), i.e.,

$J:=\left\{i \in\{1, \ldots, n\} \mid \xi_{k}^{i}<z_{k}(k=1, \ldots, s)\right\}$. If $J \in \mathcal{I}_{\mathcal{B}}^{*}$ then go to Step [6].

Step [5]: $\quad \mathcal{I}_{\mathcal{B}}^{*}:=\mathcal{I}_{\mathcal{B}}^{*} \cup\{J\}$. Calculate $\gamma_{J}$ according to Proposition 3.2.

Step [6]: $\quad$ Calculate $\alpha:=\sum_{i \in I} p_{i}$, where $I:=\left\{i \in\{1, \ldots, N\} \mid \xi_{k}^{i}<z_{k}(k=1, \ldots, s)\right\}$.

If $\alpha>\gamma^{J}$, then $\gamma^{J}:=\alpha$. Go to Step [2].

Step [7]: $\quad$ With the additional data $\mathcal{I}_{\mathcal{B}}^{*}$ and $\gamma_{J}, \gamma^{J}$ for all $J \in \mathcal{I}_{\mathcal{B}}^{*}$ solve the linear optimization problem (15).

In the following, we present some numerical results which are based on a Mathematica implementation of Algorithm 4.1. Calculations were carried out on a $1200 \mathrm{MHz}$ PC. Figure 1 shows possible reductions of a randomly generated (w.r.t. support and probabilities) 2-dimensional measure with $N=1000$ atoms. A reduction on $n=50$ atoms is considered. The left diagram of the figure illustrates the ordered solution defined in (7).

By definition, this solution selects the 50 atoms of the original measure realizing the largest probabilities. The first 49 atoms even keep the original probabilities, whereas the last one is arranged to complement the sum of theses probabilities to one in order to make the selected measure a probability measure as well. Since each of the selected 50 atoms has rather low probability whereas the set of all non-selected 950 atoms has high probability, the ordered solution places almost all mass on atom no. 50. Although such choice would be optimal in case of the closed-set discrepancy $\alpha_{\mathcal{B}_{c l}}$ according to Proposition 2.1, it is intuitively clear from the picture that such solution may not be meaningful for other discrepancy distances. Indeed, the ordered solution realizes a closed-set discrepancy of 
Figure 1: Reduction of a 2-dimensional measure with 1000 atoms (thin lines) to 50 atoms (thick lines). Heights correspond to probabilities. The left diagram illustrates the ordered solution, whereas the right diagram shows the measure which, on the fixed support of the ordered solution, is best approximating in the sense of the cell discrepancy.
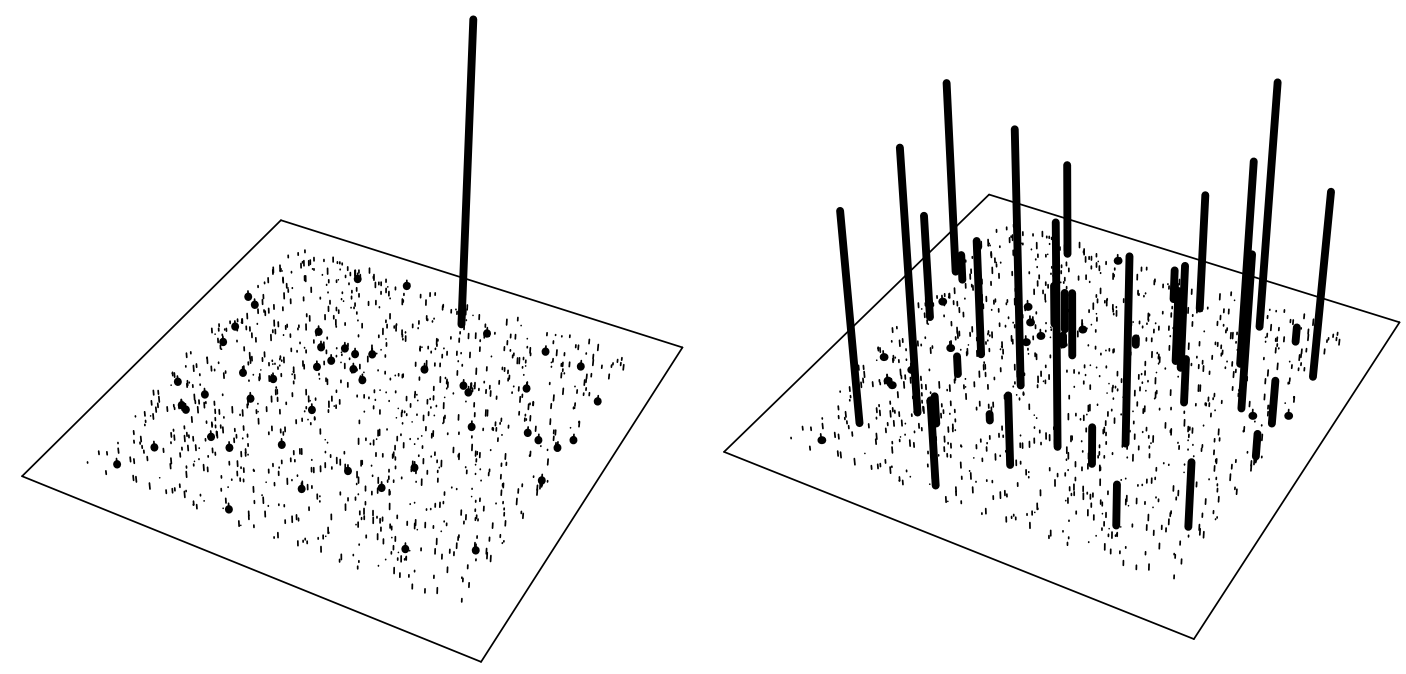

$\alpha_{\mathcal{B}_{c l}}=0.90$. Recalling, that discrepancies always take values between 0 and 1 , this value is certainly not satisfactory. It illustrates the effect of 'linear decrease' mentioned in Section 2.1: a reduction of the support to $5 \%$ of its atoms leads to a reduction of the discrepancy which is no more than $10 \%$. On the other hand, Proposition 2.1 tells us, that the same value of 0.90 is an upper bound for any solution of any other discrepancy. Now, measuring the ordered solution in the cell discrepancy instead, yields the slightly smaller value of $\alpha_{\mathcal{B}_{\text {cell }}}=0.81$, which is still far from satisfactory. However, there is no reason, why the ordered solution should be optimal with respect to the cell discrepancy, neither from the choice of the support nor from the assignment of probabilities. We illustrate this fact by keeping the same support as the ordered solution but optimizing the probabilities according to Algorithm 4.1. The resulting solution is illustrated in the right diagram of Figure 1. It realizes the cell discrepancy $\alpha_{\mathcal{B}_{\text {cell }}}=0.08$, which is optimal for the chosen fixed support (of course, even better solutions might exist for a different support). This value of $8 \%$ discrepancy obtained by $5 \%$ of the original atoms highlights the potential of Algorithm 4.1 for scenario reduction.

Numerical experiments show, that the main effort in Algorithm 4.1 is spent for the determination of supporting cells (compared to this, the time consumed by the solution of the linear program in the last step is negligible). Supporting cells are identified in this algorithm by checking all subsets of cardinality $s$ in a set of cardinality $n+s$. Therefore, the complexity of the algorithm is mainly determined by the binomial coefficient $\left(\begin{array}{c}n+s \\ s\end{array}\right)$. This suggests that the practical value of the algorithm is limited to small dimension $s$ of the random distributions and moderate cardinality $n$ of the reduced support or to small $n$ and moderate $s$ (due to the identity $\left(\begin{array}{c}n+s \\ s\end{array}\right)=\left(\begin{array}{c}n+s \\ n\end{array}\right)$ ). On the other hand, the computational 
effort is basically linear in the cardinality $N$ of the original support, so larger values of $N$ seem to be no problem.

Table 1: Computing times (in seconds) for different problem parameters (the two quantities correspond to dimensions $\mathrm{s}=3$ and $\mathrm{s}=4$, respectively.

\begin{tabular}{l|ccc} 
& $N=100$ & $N=200$ & $N=300$ \\
\hline$n=5$ & $0.24-0.49$ & $0.48-0.84$ & $0.83-1.6$ \\
$n=10$ & $0.92-2.5$ & $1.7-3.8$ & $2.9-8.3$ \\
$n=15$ & $3.2-12.1$ & $4.1-16.8$ & $9.6-33.2$
\end{tabular}

Table 1 compiles some computing times for Algorithm 4.1 under different problem sizes. As one can see, the dependence of time on the size $N$ of the original support is moderate (basically linear), whereas it quickly grows with the size $n$ of the reduced support and with the dimension $s$. This is not surprising, because the determination of all supporting cells was carried out by checking all subsets of cardinality $s$ in the set $\{1, \ldots, n+s\}$. One might wonder if there is a more efficient way of determining supporting cells than just by crude enumeration. It seems, however, that basically all mentioned subsets are potential candidates for realizing the upper bound in the cell discrepancy between two measures. Indeed, recall that the cell discrepancy coincides with the supremum distance of distribution functions (the Kolmogorov distance).

Figure 2: Difference between the distribution functions of two discrete probability measures (having 30 and 18 atoms, respectively).

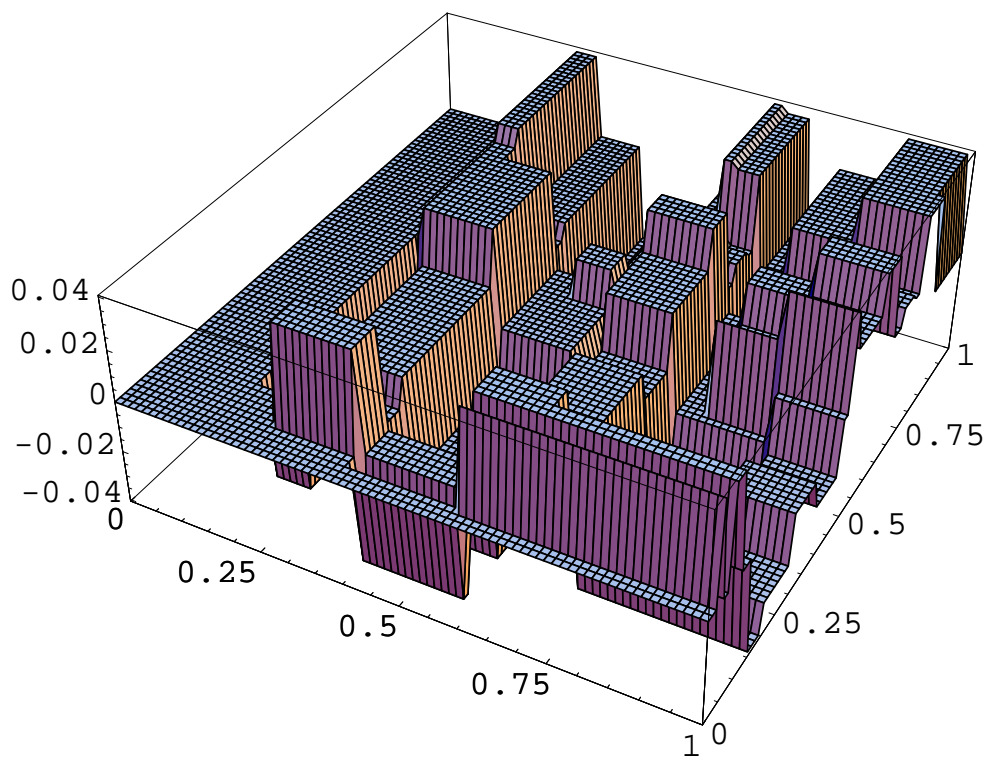


Figure 2 plots the difference of two discrete distribution functions having 30 and 18 atoms, respectively. It can be recognized at how many different regions, the maximum (positive or negative) deviation may occur.

Figure 3: Decrease of the cell discrepancy in the course of a forward selection procedure.

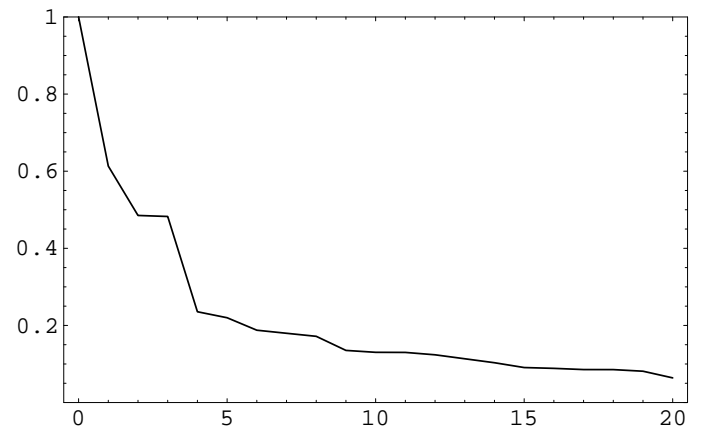

Finally, Figure 3 shows the decrease of the minimal discrepancy in the course of a forward selection procedure (see Algorithm 3.1), where the original 2-dimensional measure having 50 atoms is reduced in a stepwise manner to a measure having 20 atoms. Computation time was 160 seconds.

\section{Appendix}

Proof of Proposition 3.1. Let $J \in \mathcal{I}_{\mathcal{B}_{\text {cell }}^{*}}$ be arbitrary. By definition of $\varphi(J)$ in (13), for any $I \in \varphi(J)$ there exists some $B \in \mathcal{B}_{\text {cell }}$ such that $I=I(B)$ and $J=I(B) \cap\{1, \ldots, n\}$. Then, by definition of $I(B)$,

$$
\sum_{i \in I} p_{i}=\sum_{i \in I(B)} p_{i}=P(B)
$$

whence

$$
\begin{aligned}
\gamma^{J} & =\max _{I \in \varphi(J)} \sum_{i \in I} p_{i}=\max \left\{P(B) \mid B \in \mathcal{B}_{\text {cell }}, \quad J=I(B) \cap\{1, \ldots, n\}\right\} \\
& =\max \left\{P(B) \mid B \in \mathcal{B}_{\text {cell }}, B \cap\left\{\xi^{1}, \ldots, \xi^{n}\right\}=\cup_{j \in J}\left\{\xi^{j}\right\}\right\} \\
& =\max \left\{P([y]) \mid y \in(c, d), \quad[y] \cap\left\{\xi^{1}, \ldots, \xi^{n}\right\}=\cup_{j \in J}\left\{\xi^{j}\right\}\right\},
\end{aligned}
$$

where in the last equation it was used that the support of $P$ is contained in $(c, d)$. Let $y^{(0)}$ be a maximizing cell, i.e.,

$$
\gamma^{J}=P\left(\left[y^{(0)}\right]\right), \quad y^{(0)} \in(c, d), \quad\left[y^{(0)}\right] \cap\left\{\xi^{1}, \ldots, \xi^{n}\right\}=\cup_{j \in J}\left\{\xi^{j}\right\} .
$$

For $t \geq 0$, put $y(t):=\left(y_{1}^{(0)}+t, y_{2}^{(0)}, \ldots, y_{s}^{(0)}\right)$ and consider the enlargement $[y(t)]$ of the cell $\left[y^{(0)}\right]$ along the first coordinate direction. Put

$$
\tau:=\sup \left\{t \mid[y(t)] \cap R=\left[y^{(0)}\right] \cap R\right\} .
$$


Since the intersection of $[y(t)]$ with the finite set $R$ remains constant for small $t>0$, it follows that $\tau>0$. From $y^{(0)} \in(c, d)$, we derive that $y_{1}^{(0)}<d_{1}=r_{1}^{1}$, whence $r^{1} \notin\left[y^{(0)}\right] \cap R$. On the other hand, with $t^{*}:=d_{1}-y_{1}^{(0)}$, one gets that $r^{1} \in\left[y\left(t^{*}\right)\right] \cap R$. Consequently, $\tau \leq t^{*}<\infty$. We put $y^{(1)}:=y(\tau)$ and arrive at

$$
\begin{aligned}
{\left[y^{(1)}\right] \cap R } & =\left\{\left[y^{(1)}\right] \cap\left\{y \mid y_{1}<y_{1}^{(1)}\right\} \cap R\right\} \cup \underbrace{\left\{\left[y^{(1)}\right] \cap\left\{y \mid y_{1}=y_{1}^{(1)}\right\} \cap R\right\}}_{\alpha_{1}} \\
& =\left\{\cup_{t<\tau}[y(t)] \cap R\right\} \cup \Delta_{1}=\left\{\left[y^{(0)}\right] \cap R\right\} \cup \Delta_{1} .
\end{aligned}
$$

Once more, due to $[y(\tau+t)] \cap R$ remaining constant for small $t>0$ and to the definition of $\tau$, it follows that $\left[y^{(1)}\right] \cap R \neq\left[y^{(0)}\right] \cap R$. Consequently, $\Delta_{1} \neq \varnothing$, which means that there exists some $x^{1} \in\left[y^{(1)}\right] \cap R$ such that $x_{1}^{1}=y_{1}^{(1)}$.

Now, in the second step, we extend the construction above for the second coordinate by defining

$$
\begin{aligned}
y(t) & :=\left(y_{1}^{(1)}, y_{2}^{(1)}+t, y_{3}^{(1)}, \ldots, y_{s}^{(1)}\right) \\
\tau & :=\sup \left\{t \mid[y(t)] \cap R=\left[y^{(1)}\right] \cap R\right\} .
\end{aligned}
$$

Upon observing that $y_{2}^{(1)}=y_{2}^{(0)}<d_{2}$ and replacing $r^{1}$ by $r^{2}$, we may repeat the same argumentation as before, in order to verify that $0<\tau<\infty$, which allows to put $y^{(2)}:=$ $y(\tau)$. As before, the definition of $\tau$ allows to find that

$$
\left[y^{(2)}\right] \cap R=\left\{\left[y^{(1)}\right] \cap R\right\} \cup \Delta_{2}=\left\{\left[y^{(0)}\right] \cap R\right\} \cup \Delta_{1} \cup \Delta_{2},
$$

where

$$
\Delta_{2}:=\left\{\left[y^{(2)}\right] \cap\left\{y \mid y_{2}=y_{2}^{(2)}\right\} \cap R\right\} .
$$

With the same argument as in the first step, one infers that $\Delta_{2} \neq \varnothing$, which means that there exists some $x^{2} \in\left[y^{(2)}\right] \cap R$ such that $x_{2}^{2}=y_{2}^{(2)}$.

Continuing the construction in this way for all coordinates, we finally arrive at points $y^{(j)}$ and $x^{j}$ for $j=1, \ldots, s$, such that

$$
\begin{aligned}
{\left[y^{(j)}\right] \cap R } & =\left\{\left[y^{(0)}\right] \cap R\right\} \cup \Delta_{1} \cdots \cup \Delta_{j} \\
\Delta_{j} & =\left\{\left[y^{(j)}\right] \cap\left\{y \mid y_{j}=y_{j}^{(j)}\right\} \cap R\right\} \quad(j=1, \ldots, s)
\end{aligned}
$$

and

$$
x^{j} \in\left[y^{(j)}\right] \cap R, \quad x_{j}^{j}=y_{j}^{(j)} \quad(j=1, \ldots, s) .
$$

We put $z:=y^{(s)}$. Then,

$$
[z] \cap R=\left\{\left[y^{(0)}\right] \cap R\right\} \cup \Delta_{1} \cdots \cup \Delta_{s} .
$$


By the very construction, $\left[y^{(j)}\right] \subseteq\left[y^{(s)}\right]$ and $z_{j}=y_{j}^{(s)}=y_{j}^{(j)}$ for $j=1, \ldots, s$. Consequently,

$$
x^{j} \in[z] \cap R, \quad x_{j}^{j}=z_{j} \quad(j=1, \ldots, s),
$$

which means that $[z]$ is a supporting cell.

To prove the remaining statements of the proposition, note that the equalities $x_{j}^{j}=z_{j}$ for $x^{j} \in R$ and $j=1, \ldots, s$ imply that $c_{j} \leq z_{j} \leq d_{j}$ for $j=1, \ldots, s$ because the same bounds apply for all points of $R$. Again by construction, $y_{j}^{(j)}>y_{j}^{(j-1)}$ for $j=1, \ldots, s$, which entails that $z_{j}>y_{j}^{(0)}$ for $j=1, \ldots, s$. Now, we define a sequence of cells $\left[z^{m}\right]$ by

$$
z_{j}^{m}:=z_{j}-1 / m \quad(j=1, \ldots, s) .
$$

It follows that, for $m$ large enough, $z^{m} \in(c, d)$ and

$$
z_{j}^{m}>y_{j}^{(0)} \quad(j=1, \ldots, s) .
$$

We conclude from (24) and (23) that, for all $m \in \mathbb{N}$,

$$
\left[z^{m}\right] \cap R \subseteq[z] \cap R=\left\{\left[y^{(0)}\right] \cap R\right\} \cup \Delta_{1} \cdots \cup \Delta_{s} .
$$

The definition of $\Delta_{j}$ shows that all elements of this subset have $j$ th coordinate equal to $y_{j}^{(j)}=z_{j}$. Combining this with (24) yields

$$
\left[z^{m}\right] \cap \Delta_{j}=\varnothing \quad(j=1, \ldots, s) .
$$

Therefore, we may continue by

$$
\left[z^{m}\right] \cap R \subseteq\left[y^{(0)}\right] \cap R .
$$

On the other hand, $\left[z^{m}\right] \supseteq\left[y^{(0)}\right]$ by (25), hence $\left[z^{m}\right] \cap R=\left[y^{(0)}\right] \cap R$. We recall the fact that $y^{(0)} \in(c, d)$, whence - by definition of the set $\left\{r^{1}, \ldots, r^{s}\right\}$ in $(16)-\left[y^{(0)}\right] \cap$ $\left\{r^{1}, \ldots, r^{s}\right\}=\varnothing$. With the same reasoning, the inclusion $z^{m} \in(c, d)$ stated above, yields that $\left[z^{m}\right] \cap\left\{r^{1}, \ldots, r^{s}\right\}=\varnothing$. Owing to the definition of $y^{(0)}$, we may continue as

$$
\begin{aligned}
{\left[z^{m}\right] \cap\left\{\xi^{1}, \ldots, \xi^{n}\right\} } & =\left[z^{m}\right] \cap R=\left[y^{(0)}\right] \cap R=\left[y^{(0)}\right] \cap\left\{\xi^{1}, \ldots, \xi^{n}\right\} \\
& =\cup_{j \in J}\left\{\xi^{j}\right\} \quad(m \in \mathbb{N}) .
\end{aligned}
$$

Clearly,

$$
\operatorname{int}[z]=\cup_{m \in \mathbb{N}}\left[z^{m}\right]
$$

so (26) yields that

$$
\operatorname{int}[z] \cap\left\{\xi^{1}, \ldots, \xi^{n}\right\}=\cup_{j \in J}\left\{\xi^{j}\right\},
$$

which is (17). Finally, (22) and the inclusion $\left[z^{m}\right] \supseteq\left[y^{(0)}\right]$ lead to

$$
\gamma^{J} \geq P\left(\left[z^{m}\right]\right) \geq P\left(\left[y^{(0)}\right]\right)=\gamma^{J} \quad(m \in \mathbb{N}),
$$


hence, actually equality holds true here. Since $\left[z^{m}\right]$ is an increasing sequence of cells in the union (27), one gets that

$$
P(\text { int }[z])=P\left(\cup_{m \in \mathbb{N}}\left[z^{m}\right]\right)=\lim _{m \rightarrow \infty} P\left(\left[z^{m}\right]\right)=\gamma^{J} .
$$

Acknowledgement: This work was supported by the DFG Research Center MATHEON "Mathematics for key technologies" in Berlin and the BMBF under the grant 03SF0312E. 


\section{References}

[1] P. Billingsley, Convergence of Probability Measures, Wiley: New York, 1968.

[2] P. Billingsley and F. Topsøe, "Uniformity in weak convergence," Zeitschrift für Wahrscheinlichkeitstheorie und verwandte Gebiete vol. 7, pp. 1-16, 1967.

[3] J. Dupačová, N. Gröwe-Kuska and W. Römisch, "Scenario reduction in stochastic programming: An approach using probability metrics," Math. Program. vol. 95, pp. 493-511, 2003.

[4] H. Heitsch and W. Römisch, "Scenario reduction algorithms in stochastic programming", Comput. Optim. Appl. vol. 24, pp. 187-206, 2003.

[5] H. Heitsch and W. Römisch, "Scenario tree modelling for multistage stochastic programs," Preprint 296, DFG Research Center Matheon "Mathematics for key technologies", 2005 and submitted.

[6] H. Heitsch and W. Römisch, "A note on scenario reduction for two-stage stochastic programs," Preprint 335, DFG Research Center Matheon "Mathematics for key technologies", 2006 and submitted.

[7] R. Henrion and W. Römisch, "Metric regularity and quantitative stability in stochastic programs with probabilistic constraints," Math. Prog. vol. 84, pp. 55-88, 1999.

[8] R. Henrion and W. Römisch, "Hölder and Lipschitz stability of solution sets in programs with probabilistic constraints," Math. Prog. vol. 100, pp. 589-611, 2004.

[9] E. Hlawka, "Zur Definition der Diskrepanz," Acta arith. vol. 18, pp. 233-241, 1971.

[10] E. Hlawka and H. Niederreiter, "Diskrepanz in kompakten abelschen Gruppen I," Manuscripta Math. vol. 1, pp. 259-288, 1969.

[11] L. Kuipers and H. Niederreiter, Uniform Distribution of Sequences, Wiley: New York, 1974.

[12] P. Mück and W. Philipp, "Distances of probability measures and uniform distribution mod 1," Math. Z. vol. 142, pp. 195-202, 1975.

[13] H. Niederreiter, Random Number Generation and Quasi-Monte Carlo Methods, CBMS-NSF Regional Conference Series in Applied Mathematics, SIAM: Philadelphia, 1992.

[14] H. Niederreiter and J.M. Wills, "Diskrepanz und Distanz von Maßen bezüglich konvexer und Jordanscher Mengen," Math. Z. vol. 144, pp. 125-134, 1975.

[15] A. Prékopa, Stochastic Programming, Kluwer: Dordrecht, 1995.

[16] S.T. Rachev, Probability Metrics and the Stability of Stochastic Models, Wiley: Chichester, 1991.

[17] S.T. Rachev and W. Römisch, "Quantitative stability in stochastic programming: The method of probability metrics," Math. Oper. Res. vol. 27, pp. 792-818, 2002. 
[18] W. Römisch, "Stability of stochastic programming problems," in (A. Ruszczyński and A., Shapiro eds.) Stochastic Programming, Handbooks in Operations Research and Management Science vol. 10, pp. 483-554, Elsevier: Amsterdam, 2003.

[19] W. Römisch and R. Schultz, "Stability analysis for stochastic programs," Ann. Oper. Res. vol. 30, pp. 241-266, 1991.

[20] W. Römisch and A. Wakolbinger, "Obtaining convergence rates for approximations in stochastic programming," in (J. Guddat, H.Th. Jongen, B. Kummer and F. Nožička eds.) Parametric Optimization and Related Topics, pp. 327-343, Akademie-Verlag: Berlin, 1987.

[21] A. Ruszczyński and A. Shapiro (eds.), Stochastic Programming, Handbooks in Operations Research and Management Science, vol. 10, Elsevier: Amsterdam, 2003.

[22] R. Schultz, "Rates of convergence in stochastic programs with complete integer recourse," SIAM J. Optim. vol. 6, pp. 1138-1152, 1996. 